\title{
Two-Person Non Zero-Sum Bimatrix Game with Fuzzy Payoffs and Its Equilibrium Strategy
}

\author{
Chunyan Han, Zuofeng Gao, Yongbo Yu, Hua Zhang, Suting Zhang \& Hongxin Bai \\ College of Science, Yan Shan University, Qinhuangdao 066004, China \\ E-mail: apple-han1984@163.com
}

Fund item: Natural Science Foundation of Hebei Province

\begin{abstract}
In this paper, we consider fuzzy bimatrix games with fuzzy payoffs. Based on fuzzy max order, for such games, we define three kinds of concepts of minim ax equilibrium strategies. Some basic results obtained.
\end{abstract}

Keywords: Fuzzy bimatrix games, Fuzzy payoffs, Minimax equilibrium strategy

\section{Introduction}

Since seminal works by Neumann-Morgenstern(J.VonNeunann, 1994) and Nash(J.F.Nash., 1950 \& J.F.Nash., 1951), Game theory has played an important role in the fields of decision making theory such as economics, management, and operations research, etc. When we apply the game theory to model some practical problems which we encounter in real situations, we have to know the values of payoffs exactly. However, it is difficult to know the exact values of payoffs and we could only know the values of payoffs approximately. In such situations, it is useful to model the problems as games with fuzzy payoffs. In this case, since the expected payoffs of the game should be fuzzy-valued, there are no concepts of equilibrium strategies to be accepted widely. So, it is an important task to define the concept of equilibrium strategies and investigate their properties. In this paper, we consider fuzzy bimatrix games, namely, the games where the number of players are two and fuzzy payoffs. For such a game, we shall define three kinds of concepts of minim ax equilibrium strategies.

\section{Preliminary}

Let $R^{n}$ be n-dimensional Euclidean space, and $x=\left(x_{1}, x_{2}, \cdots, x_{n}\right)^{T} \in R^{n}$ be any vector, where $x_{i} \in R, i=$ $1,2, \cdots, n$ and $\mathrm{T}$ denotes the transpose of the vector. For any two vectors $x, y \in R^{n}$, we write $x \geq y$ if $x_{i} \geq y_{i}$, $i=1,2, \cdots, n, x>y$ and $x \neq y$, respectively.

Definition 2.1 Let $m$ be any real number and let $h$ be any positive number. A fuzzy number $\tilde{a}$ whose membership function is given by the following formula

$$
\mu_{\tilde{a}}(x)= \begin{cases}1-\left|\frac{x-m}{h}\right| & x \in[m-h, m+h] \\ 0 & x \notin[m-h, m+h]\end{cases}
$$

is called a symmetric triangular fuzzy number, and we denote the set of all symmetric triangular fuzzy numbers by $F_{T}$.

Real numbers $m$ and $h$ are called the center and the deviation parameter of $\tilde{a}$, respectively. Since any symmetric triangular fuzzy number $\tilde{a}$ is characterized by the center $m$ and the deviation parameter $h$ of $\tilde{a}$ we denote the symmetric triangular fuzzy number $\tilde{a}$ by $\tilde{a} \equiv(m, h)_{T}$.

Let $\tilde{a}$ be any fuzzy number and let $\alpha \in[0,1]$ be any real number. The set $[\tilde{a}]^{\alpha} \equiv x \in R \mid \mu_{\tilde{a}}(x) \geq \alpha$ is called the $\alpha$-level set of $\tilde{a}$. For $\alpha=0$, we set $[\tilde{a}]^{0}=\operatorname{cl}\left\{x \in R \mid \mu_{\tilde{a}}(x)>0\right\}$. where cal denotes the closure of sets. 
Since the set $[\tilde{a}]^{\alpha}$ is a closed interval for each $\alpha \in[0,1]$, we denote the $\alpha$-level set of a $\tilde{a}$ by $\left[\alpha_{\alpha}^{L}, \alpha_{\alpha}^{R}\right]$, where $a_{\alpha}^{L} \equiv \inf [\tilde{a}]^{\alpha}, a_{\alpha}^{R}=\sup [\tilde{a}]^{\alpha}$.

For any two fuzzy numbers $\tilde{a}, \tilde{b} \in F_{T}$, we introduce three kinds of binary relations.

Definition 2.2 For any symmetric triangular fuzzy numbers $\tilde{a}, \tilde{b} \in F_{T}$, we write

$$
\begin{array}{llll}
\tilde{a}=\tilde{b}, \quad \text { if }\left(a_{\alpha}^{L}, a_{\alpha}^{R}\right)^{T}=\left(b_{\alpha}^{L}, b_{\alpha}^{R}\right)^{T} & \forall \alpha \in[0,1] \\
\tilde{a} \geq \tilde{b}, \quad \text { if }\left(a_{\alpha}^{L}, a_{\alpha}^{R}\right)^{T} \geq\left(b_{\alpha}^{L}, b_{\alpha}^{R}\right)^{T} & \forall \alpha \in[0,1] \\
\tilde{a}>\tilde{b}, \quad \text { if }\left(a_{\alpha}^{L}, a_{\alpha}^{R}\right)^{T}>\left(b_{\alpha}^{L}, b_{\alpha}^{R}\right)^{T} & \forall \alpha \in[0,1]
\end{array}
$$

We call binary relations $\geq$, and $>$ a strict fuzzy max order and a strong fuzzy max order, respectively.

Theorem 2.1 let $\tilde{a}=(a, \alpha)_{T}$, and $\tilde{b}=(b, \beta)_{T}$ be any symmetric triangular fuzzy numbers. Then, it holds that

$$
\begin{array}{llll}
\tilde{a} \geq \tilde{b} & \text { If } & & |\alpha-\beta| \leq a-b \\
\tilde{a}>\tilde{b} & \text { If } & & |\alpha-\beta|<a-b
\end{array}
$$

\section{Two-person zero-sum game with fuzzy payoffs and its equilibrium strategy}

Let $I, J$ denote players and let $M=\{1,2, \cdots, m\}, N=\{1,2, \cdots, n\}$ be the sets of all pure strategies available for player $I$, and $J$, respectively. We denote the sets of all mixed strategies available for player $I$, and $J$ by

$$
\begin{gathered}
X=\left\{\left(x_{1}, x_{2}, \cdots, x_{m}\right) \in R_{+}^{m} \mid x_{i} \geq 0, i=1,2, \cdots, m, \sum_{i=1}^{m} x_{i}=1\right\} \\
Y=\left\{\left(y_{1}, y_{2}, \cdots, y_{n}\right) \in R_{+}^{n} \mid y_{j} \geq 0, j=1,2, \cdots, n, \sum_{j=1}^{n} y_{j}=1\right\}
\end{gathered}
$$

By $\tilde{a}_{i j}=\left(a_{i j}, h_{i j}\right)_{T}$, and $\tilde{b}_{i j}=\left(b_{i j}, h_{i j}^{\prime}\right)_{T}$, we denote the payoff that player $I$ receives and $J$ loses when player $I$ plays the pure strategy $i$ and player $J$ plays the pure strategy $i$. Then we have the fuzzy payoff matrix $\tilde{A}=\left(\tilde{a}_{i j}\right)_{m \times n}, \tilde{B}=\left(\tilde{b}_{i j}\right)_{m \times n}, A=\left(a_{i j}\right)_{m \times n}, H=\left(h_{i j}\right)_{m \times n}, B=\left(b_{i j}\right)_{m \times n}, H^{\prime}=\left(h_{i j}^{\prime}\right)_{m \times n}$, we call this game two-person fuzzy zero bimatrix game, and we denote it by

$$
\tilde{\Gamma}=<\{I, J\}, X, Y, \tilde{A}, \tilde{B}>
$$

Definition 3.1 A point $\left(x^{*}, y^{*}\right) \in X \times Y$ is said to be a minim ax equilibrium strategies to Game $\tilde{\Gamma}$ if it holds that

(1) $x^{T} \tilde{A} y^{*} \leq x^{* T} \tilde{A} y^{*} \quad \forall x \in X$

(2) $x^{* T} \tilde{B} y \leq x^{* T} \tilde{B} y^{*} \quad \forall y \in Y$

Definition 3.2 A point $\left(x^{*}, y^{*}\right) \in X \times Y$ us said to be a non-dominated minim ax equilibrium strategy to Game $\tilde{\Gamma}$ if it holds that

(1) There exist no $x \in X$ such that $x^{* T} \tilde{A} y^{*} \leq x^{T} \tilde{A} y^{*}$

(2) There exist no $y \in Y$ such that $x^{* T} \tilde{B} y^{*} \leq x^{* T} \tilde{B} y$

Definition 3.3 A point $\left(x^{*}, y^{*}\right) \in X \times Y$ is said to be a weak non-dominated minim ax equilibrium strategy to Game $\tilde{\Gamma}$ if it holds that

(1) There exist no $x \in X$ such that $x^{* T} \tilde{A} y^{*}<x^{T} \tilde{A} y^{*}$

(2) There exist no $y \in Y$ such that $x^{* T} \tilde{B} y^{*}<x^{* T} \tilde{B} y$

By definition, it is obvious that the following relationship holds among these definitions.

(1) If a strategy $\left(x^{*}, y^{*}\right)$ is a minim ax equilibrium strategy to Game $\tilde{\Gamma}$, it is a non-dominated minim ax equilibrium strategy.

(2)If a strategy $\left(x^{*}, y^{*}\right)$ is a non-dominated minim ax equilibrium strategy to Game $\tilde{\Gamma}$, it is a weak non-dominated minim ax strategy. 
Theorem 3.1 In order that a strategy $\left(x^{*}, y^{*}\right) \in X \times Y$ be a minim ax equilibrium strategy to Game $\tilde{\Gamma}$, it is necessary and sufficient that, for all $x \in X, y \in Y$

$$
\begin{gathered}
x^{T} A y^{*}+x^{T} H y^{*} \leq x^{* T} A y^{*}+x^{* T} H y^{*} \\
x^{T} A y^{*}-x^{T} H y^{*} \leq x^{* T} A y^{*}-x^{* T} H y^{*} \\
x^{* T} B y+x^{* T} H^{\prime} y \leq x^{* T} B y^{*}+x^{* T} H^{\prime} y^{*} \\
x^{* T} B y+x^{* T} H^{\prime} y \leq x^{* T} B y^{*}+x^{* T} H^{\prime} y^{*}
\end{gathered}
$$

Hold.

Proof. Let $\left(x^{*}, y^{*}\right) \in X \times Y$ be any minim ax equilibrium strategy to Game $\tilde{\Gamma}$. Then from Theorem 2.1, for all $x \in X, y \in Y$ we have

$$
\begin{aligned}
& \left|x^{* T} H y^{*}-x^{T} H y^{*}\right| \leq x^{* T} A y^{*}-x^{T} A y^{*} \\
& \left|x^{* T} H^{\prime} y^{*}-x^{* T} H^{\prime} y\right| \leq x^{* T} B y^{*}-x^{* T} B y
\end{aligned}
$$

By expanding and rearranging (5), we have

$$
\begin{aligned}
& x^{T} A y^{*}+x^{T} H y^{*} \leq x^{* T} A y^{*}+x^{* T} H y^{*} \\
& x^{T} A y^{*}-x^{T} H y^{*} \leq x^{* T} A y^{*}-x^{* T} H y^{*}
\end{aligned}
$$

On the other hand, by expanding and rearranging (6), we have

$$
\begin{aligned}
& x^{* T} B y+x^{* T} H^{\prime} y \leq x^{* T} B y^{*}+x^{* T} H^{\prime} y^{*} \\
& x^{* T} B y-x^{* T} H^{\prime} y \leq x^{* T} B y^{*}-x^{* T} H^{\prime} y^{*}
\end{aligned}
$$

From (7) to (10), we have (1), (2), (3), and (4).

Since

$$
\begin{aligned}
x^{* T} A_{0}^{R} y^{*} & =x^{* T}(A+H) y^{*}=x^{* T} A y^{*}+x^{* T} H y^{*} \\
x^{* T} A_{0}^{L} y^{*} & =x^{* T}(A-H) y^{*}=x^{* T} A y^{*}-x^{* T} H y^{*} \\
x^{* T} B_{0}^{R} y^{*}=x^{* T}\left(B+H^{\prime}\right) y^{*} & =x^{* T} B y^{*}+x^{* T} H^{\prime} y^{*} \\
x^{* T} B_{0}^{L} y^{*} & =x^{* T}\left(B-H^{\prime}\right) y^{*}=x^{* T} B y^{*}-x^{* T} H^{\prime} y^{*}
\end{aligned}
$$

Theorem 3.1 shows that players $I, J$ faces a pair of two-person zero-sum games with crisp payoffs $\Gamma_{1}=<$ $\{I, J\}, X, Y, A_{0}^{L}, B_{0}^{L}>, \quad \Gamma_{2}=<\{I, J\}, X, Y, A_{0}^{R}, B_{0}^{R}>$. By setting $x^{T} A y=\left(x^{T} A_{0}^{L} y, x^{T} A_{0}^{R} y\right)^{T}, x^{T} B y=$ $\left(x^{T} B_{0}^{L} y, x^{T} B_{0}^{R} y\right)^{T}$, for each $x \in X, y \in Y$, form (1), (2), (3), (4), we have $x^{T} A y^{*} \leq x^{* T} A y^{*}$, and $x^{* T} B y \leq x^{* T} B y^{*}$.

Theorem 3.2 In order that a strategy $\left(x^{*}, y^{*}\right) \in X \times Y$ be a non-dominated minim ax equilibrium strategy to Game $\tilde{\Gamma}$, it is necessary and sufficient that the following conditions holds:

(1) There is no $x \in Y$ such that $x^{* T} A y^{*} \leq x^{T} A y^{*}$ holds.

(2) There is no $y \in Y$ such that $x^{* T} B y^{*} \leq x^{* T} B y$ holds.

Proof. Let $\left(x^{*}, y^{*}\right) \in X \times Y$ be a non-dominated minim ax equilibrium strategy to Game $\tilde{\Gamma}$. First, we suppose that there exists a strategy $\bar{x} \in X$ such that $x^{* T} A y^{*} \leq \bar{x}^{T} A y^{*}$ holds. By definition, we have

$$
\left(x^{* T}(A-H) y^{*}, x^{* T}(A+H) y^{*}\right)^{T} \leq\left(\bar{x}^{T}(A-H) y^{*}, \bar{x}^{T}(A+H) y^{*}\right)^{T}
$$

By rearranging (11), we have

$$
\left(x^{* T} A y^{*}-\bar{x}^{T} A y^{*}, x^{* T} A y^{*}-\bar{x} A y^{*}\right)^{T} \leq\left(x^{* T} H y^{*}-\bar{x} H y^{*}, \bar{x}^{T} H y^{*}-x^{* T} H y^{*}\right)^{T}
$$

this implies that $x^{* T} A y^{*}<\bar{x}^{T} A y^{*}$ holds. Therefore, for all $\alpha \in[0,1]$, we have

$$
\left(x^{* T}(A-(1-\alpha) H) y^{*}, x^{* T}(A+(1-\alpha) H) y^{*}\right)^{T} \leq\left(\bar{x}^{T}(A-(1-\alpha) H) y^{*}, \bar{x}^{T}\left(A+(1-\alpha) H y^{*}\right) y^{*}\right)^{T}
$$


this implies that $x^{* T} \tilde{A} y^{*} \leq \bar{x}^{T} \tilde{A} y^{*}$ holds. This is a contradiction.

Next, we suppose that there exists a strategy $\bar{y} \in Y$ such that $x^{* T} B y^{*} \leq x^{* T} B \bar{y}$ holds. By definition, we have

$$
\left(x^{* T}\left(B-H^{\prime}\right) y^{*}, x^{* T}\left(B+H^{\prime}\right) y^{*}\right)^{T} \leq\left(x^{* T}\left(B-H^{\prime}\right) \bar{y}, x^{* T}\left(B+H^{\prime}\right) \bar{y}\right)^{T}
$$

By rearranging (12), we have

$$
\left(x^{* T} B y^{*}-x^{* T} B \bar{y}, x^{* T} B y^{*}-x^{* T} B \bar{y}\right)^{T} \leq\left(x^{* T} H^{\prime} y^{*}-x^{* T} H^{\prime} \bar{y}, x^{* T} H^{\prime} \bar{y}-x^{* T} H^{\prime} y^{*}\right)^{T}
$$

this implies that $x^{* T} B y^{*} \leq x^{* T} B \bar{y}$ holds. Therefore, for all $\alpha \in[0,1]$, we have

$$
\left(x^{* T}\left(B-(1-\alpha) H^{\prime}\right) y^{*}, x^{* T}\left(B+(1-\alpha) H^{\prime}\right) y^{*}\right)^{T} \leq\left(x^{* T}\left(B-(1-\alpha) H^{\prime}\right) \bar{y}, x^{* T}\left(B+(1-\alpha) H^{\prime}\right) \bar{y}\right)^{T}
$$

this implies that $x^{* T} \mathscr{B} y^{*} \leq x^{* T} \mathscr{B} \bar{y}$. This is a contradiction.

Conversely, let $\left(x^{*}, y^{*}\right)$ be any strategy to Game $\tilde{\Gamma}$ such that conditions (1) and (2) hold.

First, we suppose that there exists strategy $\bar{x} \in X$ such that $x^{* T} \tilde{A} y^{*} \leq \bar{x}^{T} \tilde{A} y^{*}$ holds. Then, by definition 2.2, we have

$$
\left(x^{* T}(A-H) y^{*}, x^{* T}(A+H) y^{*}\right)^{T} \leq\left(\bar{x}^{T}(A-H) y^{*}, \bar{x}^{T}(A+H) y^{*}\right)^{T}
$$

this is a contradiction.

Next, we suppose that there exists a strategy $\bar{y} \in Y$ such that $x^{* T} \tilde{B} y^{*} \leq x^{* T} \tilde{B} \bar{y}$ holds. Then, by Definition 2.2, we have

$$
\left(x^{* T}\left(B-H^{\prime}\right) y^{*}, x^{* T}\left(B+H^{\prime}\right) y^{*}\right)^{T} \leq\left(x^{* T}\left(B-H^{\prime}\right) \bar{y}, x^{* T}\left(B+H^{\prime}\right) \bar{y}\right)^{T}
$$

this is a contradiction.

By a similar way, we have the following theorem.

Theorem 3.3 In order that a strategy $\left(x^{*}, y^{*}\right) \in X \times Y$ be a weak non-dominated minim ax equilibrium strategy to Game $\tilde{\Gamma}$, it is necessary and sufficient that the following conditions hold:

(1) there is no $x \in X$ such that $x^{* T} A y^{*}<x^{T} A y^{*}$ holds.

(2) there is no $y \in Y$ such that $x^{* T} B y^{*}<x^{* T} B y$ holds

Theorem 3.4 we have the following conclusion:

(1) There is a non-dominated minim ax equilibrium strategy to Game $\tilde{\Gamma}$ at least.

(2) There is a weak non-dominated minim ax equilibrium strategy to Game $\tilde{\Gamma}$ at least.

\section{References}

D.Dubois \& H.Prade, Ranking fuzzy numbers in the setting of possibility theory, Inform. Sci. 30 (1983). 183-224.

Li Dengfeng, Fuzzy multiobjective many-person decision makings and games, Beijing, National Defense Industrial University Press. (2002).

L.Campos, Fuzzy linear programming models to solve fuzzy matrix games. Fuzzy Sets and Systems 32. (1989). 275-289.

J.F.Nash, Equilibrium points in n-person games, Proc. Nat. Acad. Sci. 36. (1950). 48-49.

J.F.Nash, Noncooperative games, Ann. Of Math.54. (1951). 286-295.

J.P.Aubin, Mathematical methods of games and economic theory, North-Holland. Amsterdam. (1979).

J.P.Aubin, Cooperative fuzzy game. Math. Oper. Res. 6 (1984). 1-13.

J.VonNeunann \& O.Morgenstern, Theory of games and economic behavior, Princeton University Press. Princeton. NJ, (1944).

Tan Chunqiao \& Zhang Qiang, The general two-person zero-sum game, Fuzzy systems and mathematics. (2006). 
T.Maeda, On characterization of equilibrium strategy of two-person zero-sum games with fuzzy payoffs. Fuzzy Sets and Systems, (2003). (139): 283-296.

T.Maeda, Characterization of the equilibrium strategy of the bimatrix game with fuzzy payoff. Journal of Mathematical Analysis and Applications. (2000). (251):885-896.

V.Vijay, S.Chandra \& C.R.Bector, Matrix games with fuzzy goals and fuzzy payoffs, (2005). (33): 425-429. 